\title{
A Multimode Immersive Conceptual Design System for Architectural Modeling and Lighting
}

\author{
Marcio Cabral* $^{*}$ Peter Vangorp Gaurav Chaurasia Emmanuelle Chapoulie \\ REVES/INRIA Sophia Antipolis \\ George Drettakis \\ REVES/INRIA Sophia Antipolis
}

Martin Hachet

IPARLA/INRIA Bordeaux

\begin{abstract}
We present a new immersive system which allows initial conceptual design of simple architectural models, including lighting. Our system allows the manipulation of simple elements such as windows, doors and rooms while the overall model is automatically adjusted to the manipulation. The system runs on a four-sided stereoscopic, head-tracked immersive display. We also provide simple lighting design capabilities, with an abstract representation of sunlight and its effects when shining through a window. Our system provides three different modes of interaction, a miniature-model table mode, a fullscale immersive mode and a combination of table and immersive which we call mixed mode. We performed an initial pilot user test to evaluate the relative merits of each mode for a set of basic tasks such as resizing and moving windows or walls, and a basic light-matching task. The study indicates that users appreciated the immersive nature of the system, and found interaction to be natural and pleasant. In addition, the results indicate that the mean performance times seem quite similar in the different modes, opening up the possibility for their combined usage for effective immersive modeling systems for novice users.
\end{abstract}

\section{INTRODUCTION}

Creating and manipulating 3D models is one of the hardest tasks in virtual environments. Traditional 3D modeling packages such as the Autodesk Maya and Blender have a very high learning curve, and are inappropriate for fast conceptual design by lay users, especially in immersive settings. Recently several research [6, 12] and commercial tools, such as Google's SketchUp, efforts have concentrated on providing intuitive and easy-to-use interfaces to build simple 3D models. These results and products are an indication of the widespread interest for such functionality. Intuitively, the creation of 3D models should be an ideal candidate for true 3D interfaces, since the content is inherently 3D. Such considerations have inspired futuristic artistic work ${ }^{1}$ in which a 3D gestural interface is used to create a complex and visually rich environment.

Based on this intuition, our goal is to study direct manipulation for basic 3D modeling in an immersive setting, in the context of conceptual or initial design for architecture. An example of a system allowing such conceptual design is the work of Cabral et al. [6], which allows users to manipulate geometry and texture directly, and automatically updates the model to respond to these changes. In addition to geometry and texture, we are interested in the manipulation of lighting, which is an important element in modeling and design, especially for architectural models. The last element of interest is the interaction paradigm and in particular the "view" or "mode" in which the user performs the modeling task: egocentric

*e-mail: Firstname.Lastname@inria.fr

http://www.branitvfx.com/worldbuilder/
(1:1 scale) immersive mode, world-in-miniature or exocentric mode or a combination of both (see Fig. 1).

The goals of this study are to create a prototype system in an immersive setting, which supports casual modeling of textured geometry and lighting, and to investigate the relative merits of the different view modes with a pilot user study. Our system is designed to be simple and easy to learn, targeting novice users. Our main contribution is to incorporate the use of an automated geometry and texture manipulation system, including basic realtime lighting design in an immersive, WIM-like interface setting.

\section{Previous WORK}

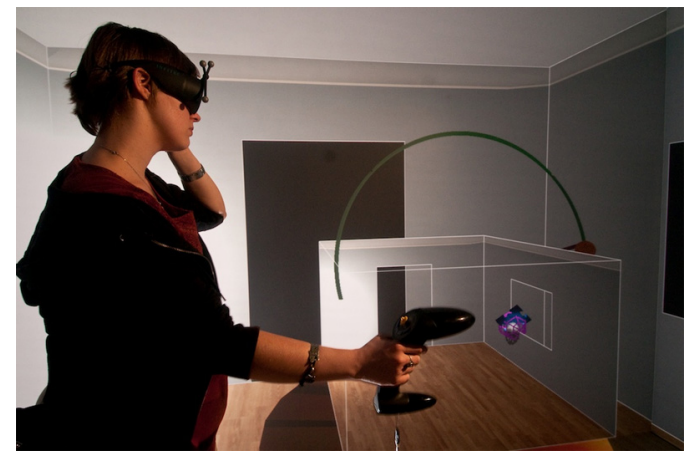

Figure 1: A view of our system in "mixed mode" where a miniature and immersive version are combined. INRIA / Photo Kaksonen.

In architectural design, users need tools for interaction which are simple yet provide enough flexibility to achieve the desired goal. Architectural design and geometric modeling have traditionally been priviledged applications for virtual reality systems [8, 5] 14]. Our approach draws inspiration from these and others but addresses the problem from a different perspective. The closest previous work is the World-in-Miniature (WIM) system [19] and derivations [13]. They presented a miniature version mixed with a scale 1:1 model (equivalent to our "mixed mode"), but for simple manipulations of viewpoint and of the virtual scene. In contrast, we base our work on a system [6] which provides automatic assistance in the context of a "quick prototype" modeling or conceptual design application [1]. More sophisticated modeling/design operations can thus be performed. In addition, we study lighting design in this context, using a simple "one-bounce" global illumination approximation to evaluate the consequences of light source changes in an immersive setting.

By targeting specific developments to the universal 3D tasks of manipulation, selection, system control, navigation and symbolic input [3] we can focus on providing a resourceful experience for architectural environment design. Our study is related to several aspects of manipulation of objects in large scale and immersive displays; these are usually accomplished with some degree of physical navigation and interaction. 
The idea of automatically scaling an object and placing it within reach in front of the user has been proposed by Mine et al. [16] and makes use of the concept of proprioception.

We use a simple form of bimanual interaction for resizing (similar to that used on 2D touch-sensitive devices). In previous work, bimanual interfaces have been used in immersive settings [2]. We try to follow ideas which were initially observed by Guiard [11 regarding the usage of both hands in our resizing gesture.

In our system, the user may switch between an immersive, scale $1: 1$ view and a world-in-miniature view, which implies a viewpoint change. Ware and Osborne proposed a technique [20] called Eyeball in Hand in which the user directly controls the viewpoint with a device that directly maps its position and orientation. Our approach adopts the use of widgets to disambiguate rotation in all axes and provide the user more precise control. Go-Go [17] and PRISM [9] switch between direct (1:1) and scaled interaction, either for largescale or precise manipulation, based on the user's intentions which are determined by the velocity of the hand. The HOMER system [4] attaches a virtual hand to an object selected by a light ray selection, allowing the user to manipulate far objects with no extra work.

Lucas et al [15] introduce alternative 3D resizing widgets, including the straightforward generalization of the familiar 2D resizing widgets, the Pointer Orientation based Resize Technique (PORT), and the Gaze-Hand resize technique. Users prefer the familiar widgets, but after a training period they perform faster with the novel widgets.

\section{Prototype System Description}

We designed and implemented a system for 3D immersive modeling aimed at initial prototyping or conceptual design, including modification of textured geometry and lighting.

The underlying modeling system is based on [6], in which the user "fixes" a set of vertices, and then manipulates one or more "variable" vertices. The system solves an optimization and updates the rest of the model accordingly. Different "model pieces" can be assembled, which communicate through portals allowing the propagation of the mesh modifications.

For our prototype, we use a simplified version of the system in [6] to deal with the specific task of building a simple house from a set of pre-built rooms which can be assembled. The user can add doors and windows which can subsequently be manipulated. Compared to [6] we have added new functionality for lighting and the control of sun position, which we describe later. Our system is interactive, and thus geometry and textures of 3D models, as well as lighting, are updated on the fly.

The system receives input from the user through the use of a bimanual interface, which is currently implemented using an ART 2 wand (dominant hand) and a hand-tracking device (non-dominant hand). The user is head-tracked and all interaction is performed in a BARCO iSpace $3.2 \times 3.2 \times 2.4 \mathrm{~m}$ 4-wall system 3 powered by a QuadroPlex NVidia card. The system was developed in $\mathrm{C}++$ /OpenGL using in house libraries.

The system is best understood by watching the accompanying video which presents a comprehensive view of its usage.

\subsection{Widgets and Modes}

The system supports three different interaction modes, notably table, immersive (1:1 scale, fully immersive) and mixed, which is a combination of the former two modes. The user can interact with the environment through a set of "selectable widgets". Our goal is to provide visual feedback to the user indicating which actions are available at a given point in space. Although widgets introduce an extra level of abstraction [7], we believe that in an architectural

\footnotetext{
${ }^{2} \mathrm{http}: / /$ www.ar-tracking.de/

${ }^{3}$ http://www.barco.com/en/virtualreality/product/732.
}

environment widgets clearly indicate which actions are available to the user at a given location.

Each widget allows the user to perform a unique action. For instance, a "wall widget" allows the user to grab, then move or resize the wall (Fig.2(a) and (b)). Alternatively, a "window widget" allows the user to resize or move a window within the limits of a wall (Fig. 2.c) and (d)). A "human avatar" can be dragged and dropped inside a particular room, changing the viewpoint so that the user is transported to immersive mode inside that room (Fig. 2 eg)); a button-press is used to go back. Other mode changes are also accomplished through a button-press on the wand. The list of available widgets are: wall, window, sun widget, add door, add window, connect room, go into immersive mode.

Actions can be executed in three different editing modes. All three modes take advantage of the immersive nature of a iSpace system, putting the user within reach of all tools for prototyping an architectural model. All modes allow co-located interaction based on the widgets described above.

Table Mode The first mode is based on a table top design, with building blocks and tools available for interaction: the user stands in front of a table with the building blocks available for usage (Fig. 3 a)). The user begins the architectural design by choosing a pre-designed 3D model e.g., a room, corridor, etc. Room selection is provided to the user with a floating rotation menu with options, displayed to his right and within reach (co-located paradigm). (Fig. 3 b). As the user waves his hand over the menu, it rotates and displays all available options; the user presses the wand button to choose the appropriate room (please see video).

Table (and mixed) mode resembles the WIM paradigm, with the addition of the underlying table which gives the user stability and a "real location" to position its prototype. Since the user observes the world from an external perspective, this is an exocentric view.

Immersive Mode The second editing mode is egocentric: the world is displayed at a 1:1 scale and the user is correctly immersed in the architectural model (Fig. 3(c)). In this second mode the user has the same editing tools available as in the previous mode. Interaction occurs in the same manner as in table mode using the widgets. One of the challenges in this particular mode is navigation: the user is free to walk around in the confined space of the iSpace system to explore and edit the model. This also translates to a 1:1 scale translation inside the virtual environment. However, it is often the case that the VR environment is bigger than the actual physical iSpace room. To overcome this limitation, the user can "fly through" the environment using the flystick.

Mixed mode The third editing mode is a combination of the two previous editing modes. The user is positioned at a $1: 1$ scale inside the 3D model. While in this mode, the user is able to visualize a small scale version of the model in front of him.

\subsection{Lighting Design}

A special widget is provided for lighting design: the user can move an orange colored and textured sphere representing the sun over two trajectories (latitude and longitude). The date and time are displayed in 3D next to the sun widget; when these are changed the lighting is updated in the scene (see Figure 2 $h$ ) and (i)).

A simplified one-bounce illumination model is used: a directional light source illuminates the scene through the windows in the room created by the user. The center of the projection of the window onto the floor is used as a secondary light source to update the illumination of the walls in the room, i.e., using a point to point differential form-factor [10]. Since we compute one bounce illumination and assume that all points are visible to the sun patch $P$, the form factor is computed per pixel on the GPU using the following formula: 


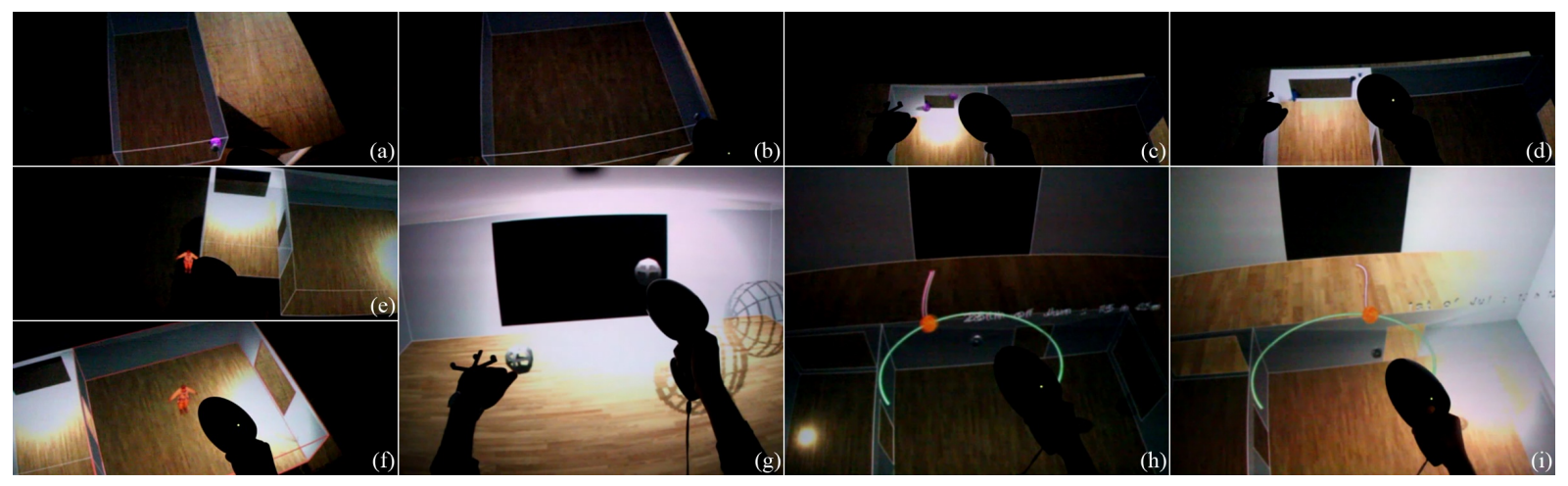

Figure 2: (a) The wall widget can be grabbed, resulting in a resized wall (b). (c) A window widget allows move and resize (bimanual) shown in (d). (e) The user selects the "human avatar" on the left and drags it to a location in the room (f). The user is then transported to that point in 1:1 scale $(\mathrm{g})$. (h) The sun widget allows manipulation of the location of the sun (i), resultsing in an update of one-bounce illumination.

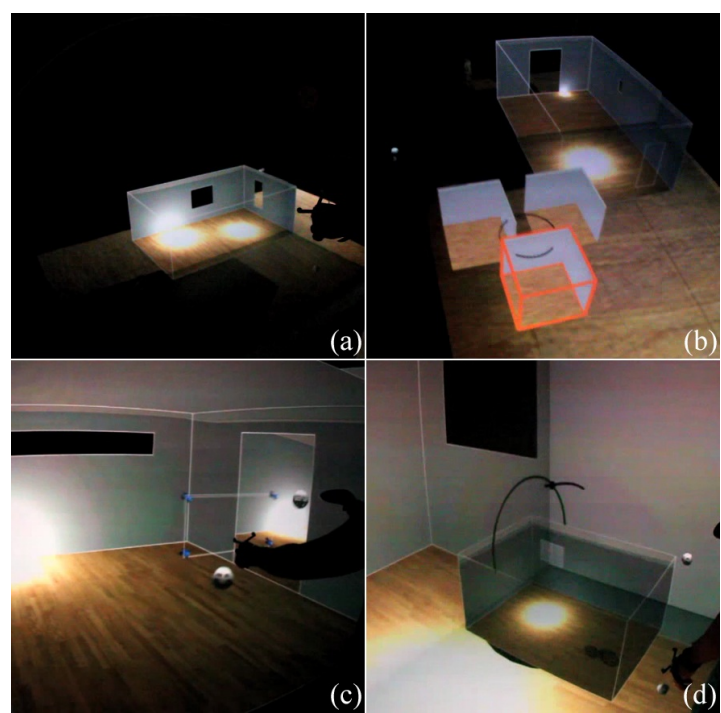

Figure 3: (a) Table mode, (b) Room rotational menu (c) Immersive mode (d) Mixed mode

$$
F_{x, P}=\frac{\cos \theta \cos \theta^{\prime}}{\pi r^{2} \cdot A_{P}}
$$

For a sun patch $P$, we integrate over all points $x$ in the room, where $r$ is the distance between each point $x$ and $P, \theta$ and $\theta^{\prime}$ are the angles between the line connecting $P$ and $x$ and $A_{P}$ is the area of the projected sun patch $P$ on the surface of the room. This is a simple approximation, but provides an effective first impression of global illumination changes due to modifications in lighting.

\section{USER STUDIES AND EVALUATION}

The integration of the prototype modeling system of [6] and a simple lighting control interface into an immersive interface allows us to study the relative effectiveness of the different modes (table, immersive and mixed) for several different tasks. Although the results are preliminary, it provides useful indication of what would be interesting to study in detail. Details on the user study procedure and basic statistical analysis are included in the supplemental material.

The study has two parts: a first set of specific tasks and then a more open task in which the user is asked to construct a three room house. The specific tasks are: resize a wall, add/move/resize a window, and move the sun. For placement and resize tasks, the user is presented with a target guide in wireframe which allows objective error in the task to be measured. For the sun task, the user is presented with a square having a target intensity, and is asked to move the sun to match the target intensity (see video).

The following table gives an overview of which actions can be accomplished in which modes:

\begin{tabular}{lccc} 
& Table & Immersive & Mixed \\
\hline Add window & $\checkmark$ & & \\
Move window & $\checkmark$ & & \\
Resize window & $\checkmark$ & & \\
Resize wall & $\checkmark$ & $\checkmark$ & \\
Add door & $\checkmark$ & $x$ & \\
Add room & $\checkmark$ & $x$ & \\
Move sun & $\checkmark$ &
\end{tabular}

The participants are also presented with a questionnaire with subjective questions after the experiment.

\subsection{Experimental procedure}

The experiment starts with a training session so the participant can learn the interface. The participant is introduced to the interface by an experimenter who guides the user through the process. The participant starts in table mode, with a simple room created and is then guided through each action available in each mode. The participant is then asked to perform the action until the experimenter is satisfied that the knowledge of the action has been acquired. The training session takes between 10-15 minutes.

After a short break, the participant is asked to perform the set of tests involving the actions listed above. The window and sun tests are performed for each of Table, Immersive and Mixed mode; the wall resize test is only performed in Table and Mixed mode (see table above). The user completes each one of the tasks in a single mode; the user is not allowed to change modes in this part of the experiment.

For all add/move/resize tests, we record time to achieve the task and error compared to the wireframe target. Completion time is also recorded for the sun test and the error in color between the target square and the corresponding square on the wall is computed (see Fig. 4 second row).

For the open task, the participants were instructed to construct a three room house within a time limit of 5 minutes and to make sure that all rooms have enough sunlight in winter but not too much in summer. In this part of the experiment, the user can freely change between interaction modes.

A total of 8 participants completed the study in the iSpace of our institute, all male with ages varying from 25 to 40 . All reported normal vision except for one subject who had stereo deficiency. 


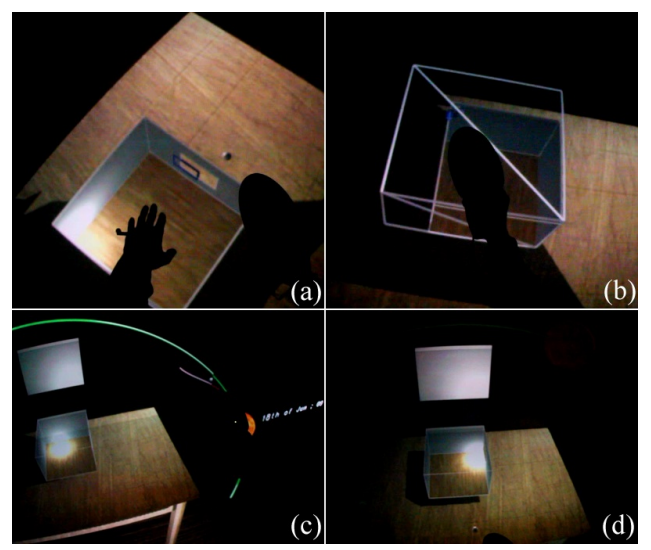

Figure 4: (a) Target wireframe for a window resize and (b) a room resize task. (c) Sun target in table mode (start of test) (d) Sun target at the end of the test.

\subsection{Experimental Results}

Tasks in the immersive mode can take slightly longer if the user first needs to walk, virtually or physically, to get closer to the object. Some participants reported that it was difficult to reach the corners of the large windows when they were standing too far away. On the other hand resizing the windows from a suitable location was a little more accurate in immersive mode than in the other modes.

The widgets and the selection sphere that floated just in front of the flystick cast a shadow on nearby surfaces. For widgets in the house, participants could use these shadows as a depth cue to guide them to the correct position. Most participants initially had some difficulty selecting the sun widget because there was no shadow to guide them and they had to rely on the stereoscopic depth cue.

During the 5 minutes of the open task, participants switched between modes on average 13 times, suggesting that they preferred to perform some operations in specific modes. Most editing was performed in table mode, while immersive or mixed mode were used mainly for inspection and even for fun. Participants used the undo functionality on average only twice since any move or resize operations could easily be corrected manually.

The responses to the questionnaire were all positive about the system overall, finding the experience to be interesting and engaging. There was a small preference for table mode (4 subjects). Overall users appreciated the immersive nature of the system, and found interaction to be natural and pleasant.

\section{Discussion and Conclusion}

The results of the pilot study tend to indicate that none of the modes we used demonstrates clear superiority for the tasks proposed. As a result, all modes can be potentially used, depending on user preference, level of immersion required etc. The results also indicate that measured performance times are similar in table and mixed mode. This is interesting since in mixed mode the effects of lighting on the overall environment can be appreciated much better than in table mode only. Given the slight preference for table mode, it may be the case that mixed mode can be used as the "default view", but the user can "switch off" immersive view when they wish to concentrate on a specific task in table mode. Additional study is required to answer this question, with more complex tasks.

In conclusion, we have presented a first immersive system which allows simple conceptual design for textured geometric models and basic lighting design. We implemented and tested this system in an immersive room with a four-sided display, using three different modes: table, immersive (1:1 scale) and mixed, which is a combination of the first two (WIM). When asked to perform a more open task, all modes were used, and novice users all mentioned that they found the experience rewarding and interesting.
We are interested in pursuing these ideas further in the future, and notably investigating more complex modeling and lighting tasks, making the system appropriate for expert users and architects. It is particularly interesting to investigate the use of sophisticated global illumination in the context of lighting design, and the importance of different levels of realism, in the spirit of [18].

\section{ACKNOWLEDGEMENTS}

We acknowledge the generous support of NVIDIA (Professor Partnership Program), and the Autodesk Research Donation program. This research was partially funded by the ARC NIEVE collaborative project (http://www-sop.inria.fr/reves/NIEVE) and the Regional Council of Provence-Alpes-Côte d'Azur.

\section{RefERENCES}

[1] L. Anderson, J. Esser, and V. Interrante. A virtual environment for conceptual design in architecture. In EGVE'03, 2003.

[2] R. Balakrishnan and G. Kurtenbach. Exploring bimanual camera control and object manipulation in $3 \mathrm{~d}$ graphics interfaces. In $\mathrm{CHI}$ ' 99 . ACM, 1999.

[3] D. A. Bowman, J. L. Gabbard, and D. Hix. A survey of usability evaluation in virtual environments: Classification and comparison of methods. Presence: Teleoperators and Virtual Environments, 11, 2002.

[4] D. A. Bowman and L. F. Hodges. An evaluation of techniques for grabbing and manipulating remote objects in immersive virtual environments. In $I 3 D^{\prime}$ '97. ACM, 1997.

[5] H.-J. Bullinger, W. Bauer, G. Wenzel, and R. Blach. Towards user centred design (ucd) in architecture based on immersive virtual environments. Computers in Industry, 61(4), 2010.

[6] M. Cabral, S. Lefebvre, C. Dachsbacher, and G. Drettakis. Structure preserving reshape for textured architectural scenes. CGF(Eurographics), 2009.

[7] J. Chen, D. A. Bowman, and D. H. Laidlaw. Poster: A hybrid direct visual editing method for architectural massing study in virtual environments. 3DUI, 2009.

[8] J. Deisinger, R. Blach, G. Wesche, R. Breining, and A. Simon. Towards immersive modeling - challenges and recommendations: A workshop analyzing the needs of designers. In EGVE' 00 , pages 145156, 2000.

[9] S. Frees and G. D. Kessler. Precise and rapid interaction through scaled manipulation in immersive virtual environments. In $V R{ }^{\prime} 05$, Washington, DC, USA, 2005. IEEE Computer Society.

[10] D. Greenberg, M. Cohen, and K. Torrance. Radiosity: A method for computing global illumination. The Visual Computer, 1986.

[11] Y. Guiard. Asymmetric division of labor in human skilled bimanual action: The kinematic chain as a model. Journal of Motor Behavior, 19, 1987.

[12] T. Igarashi, S. Matsuoka, and H. Tanaka. Teddy: a sketching interface for $3 \mathrm{~d}$ freeform design. In SIGGRAPH Courses. ACM, 2006.

[13] J. J. LaViola, Jr., D. A. Feliz, D. F. Keefe, and R. C. Zeleznik. Handsfree multi-scale navigation in virtual environments. I3D ' 01 , New York, NY, USA, 2001. ACM.

[14] J. Leigh and A. Johnson. Calvin: an immersimedia design environment utilizing heterogeneous perspectives. IEEE ICMCS '96.

[15] J. F. Lucas, J.-S. Kim, and D. A. Bowman. Resizing beyond widgets: object resizing techniques for immersive virtual environments. In $\mathrm{CHI}$ '05. ACM, 2005.

[16] M. R. Mine, F. P. Brooks, Jr., and C. H. Sequin. Moving objects in space: exploiting proprioception in virtual-environment interaction. In SIGGRAPH '97. ACM Press/Addison-Wesley Publishing Co., 1997.

[17] I. Poupyrev, M. Billinghurst, S. Weghorst, and T. Ichikawa. The go-go interaction technique: non-linear mapping for direct manipulation in vr. In UIST'96. ACM, 1996.

[18] M. Slater, P. Khanna, J. Mortensen, and I. Yu. Visual realism enhances realistic response in an immersive virtual environment. IEEE Computer Graphics and Applications, 29, 2009.

[19] R. Stoakley, M. J. Conway, and R. Pausch. Virtual reality on a WIM: interactive worlds in miniature. In CHI'95, 1995.

[20] C. Ware and S. Osborne. Exploration and virtual camera control in virtual three dimensional environments. In I3D '90. ACM, 1990. 